\title{
BMJ Open Multicentre prospective randomised single-blind controlled study protocol of the effect of an additional parent- administered sensorimotor stimulation on neurological development of preterm infants: Primebrain
}

Karine Pelc, ${ }^{1,2}$ Irit Daniel, ${ }^{2}$ Bernard Wenderickx, ${ }^{2}$ Bernard Dan, ${ }^{2,3}$ on behalf of the Primebrain group

To cite: Pelc K, Daniel I, Wenderickx B, et al. Multicentre prospective randomised single-blind controlled study protocol of the effect of an additional parentadministered sensorimotor stimulation on neurological development of preterm infants: Primebrain. BMJ Open 2017;7:e018084. doi:10.1136/ bmjopen-2017-018084

- Prepublication history for this paper is available online. To view these files, please visit the journal online (http://dx.doi. org/10.1136/bmjopen-2017018084).

Received 5 June 2017 Revised 10 0ctober 2017 Accepted 11 October 2017

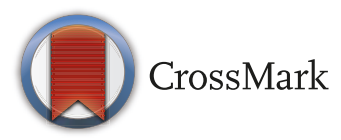

${ }^{1}$ Department of Paediatrics, Centre Hospitalier Universitaire Saint-Pierre, Université libre de Bruxelles (ULB), Brussels, Belgium

${ }^{2}$ Department of Neurology, Hôpital Universitaire des Enfants Reine Fabiola, Université libre de Bruxelles (ULB), Brussels, Belgium

${ }^{3}$ Inkendaal Rehabilitation Hospital, Vlezenbeek, Belgium

Correspondence to Dr Karine Pelc;

karine.pelc@ulb.ac.be

\section{ABSTRACT}

Introduction Preterm and very low birthweight infants are at increased risk for neurodevelopmental disorders, including cerebral palsy, sensory impairment and intellectual disability. Several early intervention approaches have been designed in the hope of optimising neurological development in this context. It seems important that the intervention takes into account parental mental health, focuses on parent-child interactions and lasts sufficiently long. This study aims to evaluate the effects of a stimulation programme administered by parents until 6 months post-term on motor and neurophysiological development of infants born preterm.

Methods and analysis Participants will be infants born $<32$ weeks' gestation and/or with a birth weight $<1500 \mathrm{~g}$ recruited prospectively from two tertiary neonatal intensive care units. They will be randomly assigned to receive nationally recommended follow-up only (control group) or also a stimulation programme between 37 weeks' gestation and 6 months' corrected age. Perinatal, clinical neurodevelopmental, sociodemographic and neuroimaging (ultrasonography or MRI) data will be collected. Bayley Scales of Infant Development will be used up to 24 months' corrected age and Parental Stress Index at 6, 12, 18 and 24 months' corrected age. High-density (64 or 128 electrodes) EEG, visual, somatosensory and long latency auditory evoked potentials will be recorded at term age, 3, 6, 12, 18 and 24 months' corrected age. They will be analysed for spatiotemporal frequency bands contents and source localisation.

Ethics and dissemination The study was approved by the Ethics Committees of the Hôpital Universitaire des Enfants Reine Fabiola and CHU Saint-Pierre. Results dissemination will be made for stakeholders and families, reports will be written for parents, healthcare providers and policymakers, and scientific papers will be published.

Trial registration number NCT02159534; Pre-results.
Strengths and limitations of this study

- Intervention with direct clinical applicability.

- Clinical and neurophysiological Iongitudinal assessment.

- High-density EEG recording technique.

- No term-born infant group for comparison.

- Variability related to parent-administered intervention.

\section{INTRODUCTION}

\section{Background and rationale}

Preterm birth and/or low birth weight are recognised risk factors for motor, cognitive and behavioural impairment in the developing child. ${ }^{1-5}$ A number of early intervention programmes based on sensorimotor stimulation have been designed in the hope of improving these children's neurological development. Early intervention may be contemplated based on the relatively high plasticity of the developing brain in young infants, when neural multiplication and migration have been mostly completed and synaptogenesis is very active. ${ }^{6}$ High brain plasticity is observed from about 2 months before term to about 6 months after term age. A particular emphasis has been placed on activity-dependent plasticity. ${ }^{7} 8$ The suggested early intervention programmes show marked heterogeneity in type of stimulation, age at which it is offered, duration and frequency, and studies to evaluate them also show great variation in assessment tools and follow-up. ${ }^{9}$ As a result, the effect of early intervention on development remains unclear, which constitutes an important gap 
of knowledge. Recent meta-analysis showed a positive influence of some early preterm infants intervention programmes on cognitive and motor outcomes during infancy and also cognitive benefits persisting into preschool age. ${ }^{9}$ The authors emphasised the need to determine which early developmental interventions are effective in improving motor and cognitive outcomes. This should rely on sound approaches of functional evaluation. Recently, high-density EEG (HD-EEG) has been shown to bring out rhythmic synchronous activity and transient events including those elicited by afferent stimuli in infants. ${ }^{10}$ Primebrain is a parent-administered early intervention programme focusing on motor control and social interaction, ${ }^{11}$ which should be soundly evaluated. It provides stimulation over 6 months during a particularly sensitive period for activity-dependent plasticity. ${ }^{6812}$

\section{Hypotheses/objectives}

The study population consists of children born before 32 weeks' gestation and/or with a birth weight $<1500 \mathrm{~g}$ because these are important risk factors of neurodevelopmental disorders. ${ }^{1-5}$ This study evaluates the effect of the Primebrain early stimulation programme, whose items were selected according to a Delphi process through five subsequent rounds leading to consensus between experts in physical therapy, neuroscience, neonatal care and developmental neurology. ${ }^{11} \mathrm{~A}$ feasibility study was conducted on 15 infants born before 32 weeks of gestation. ${ }^{11}$ It led to identification of strengths of the programme including home intervention, weaknesses including time-consuming involvement, opportunities such as possible developmental effect and threats such as compliance difficulty. Parents' individual support was then planned in the Primebrain programme and organised on a regular basis. Systematic reviews stress the need to evaluate the effect of this type of approach on the development of at-risk children. ${ }^{9}$ Bayley Scales of Infant Development (BSID-III) is most often used to characterise the development of children in this age group.

The research is based on the notion of critical periods for brain plasticity, ${ }^{8}$ which is particularly intense for the cortico-spinal tract up to 6 months post-term, ${ }^{12}$ and the importance of parents' involvement in the care of children at risk. ${ }^{9}$

\section{Primary research question}

In children born very preterm or with a very low birth weight, is an additional parent-administered programme (Primebrain) compared with the absence of specific stimulation in the context of systematic monitoring as organised in Belgium associated with improvement of the neurological development assessed using BSID-III at 24 months' corrected age?

\section{Secondary research questions}

In the same population: (1) Is there a developmental trend as measure by BSID-III at 6,12 and 18 months' corrected age? (2) Is the additional parent-administered programme (Primebrain) associated with decreased parental stress assessed using Parental Stress Index (PSI) at 6,12, 18 and 24 months' corrected age compared with the systematic monitoring as organised in Belgium? (3) Do patterns of spatial organisation of the oscillatory brain activities and of evoked potentials emerge during the first 24 months of life based on HD-EEG recording?

\section{Trial design}

This is a multicentric prospective randomised controlled clinical study. The parents are not blinded; BSID-III, PSI and neurophysiological data assessors are blinded.

\section{Registration}

The study was registered on Clinicaltrials.gov (NCT02159534), V.3.0, updated on 9 June 2017.

\section{METHODS AND ANALYSIS}

The SPIRIT guidelines were followed (www.equatornetwork.org/reporting-guidelines/spirit-2013-statementdefining-standard-protocol-items-for-clinical-trials/) .

\section{Participants}

The cohort is being recruited from the neonatal intensive care units of two university hospitals in Brussels from the same university (Université libre de Bruxelles) and the same hospital group (CHU Bruxelles), Hôpital Universitaire des Enfants Reine Fabiola and CHU Saint-Pierre. They are located in Brussels, $9 \mathrm{~km}$ apart, functioning in the same way and have similar recruitment. The very preterm infants are inborn in both settings.

Inclusion criteria are infants born before 32 weeks of gestation or with a birth weight $<1500 \mathrm{~g}$. Gestational age is defined as the time between the first day of the last menstrual period and the day of the birth. Corrected age is calculated by subtracting the number of weeks of prematurity from the chronological age; corrected age is expressed in months. Infants with severe congenital malformation (cerebral, cardiac or within a syndrome), neurodevelopmental genetic syndrome, cytomegalovirus, rubella or toxoplasmosis congenital infection, neonatal hypothyroidism, peripheral neurological disorder or congenital muscular disorder, or neurodegenerative disorder, are excluded. If one or more of these exclusion criteria are diagnosed during the course of the study, the participant will leave the study.

Infants / parents dyads are recruited during the neonatal stay. In the studied settings, infants born very preterm stay in the neonatal department until around term age. Recruitment takes place when their condition does not require intensive care any more, typically around 34 weeks of gestation.

Perinatal, clinical neurodevelopmental, socio-demographic and neuroimaging (ultrasonography or MRI) data will be collected. The social risk will be evaluated using the Social Risk Index, ${ }^{13}$ which takes into account 
the family structure, maternal age at birth, education of the primary caregiver, occupation and status of the primary income earner, and language spoken at home. At least ultrasonography is expected to be available for all infants; MRI for many, at term age, as this is common practice for very preterm in the service level of the participating hospitals. For the study, the last brain ultrasonography performed during the neonatal stay is collected. Intraventricular haemorrhage is classified from grade I to IV according to Volpe. ${ }^{14}$ Brain MRI abnormalities at term age will be classified and scored in white matter abnormalities and grey matter abnormalities according to Woodward et al..$^{15}$ Supportive analyses will take those into account when comparing the general characteristics of the study and comparison groups.

\section{Intervention}

Infants in the experimental group receive the Primebrain sensorimotor stimulation programme. The programme aims to facilitate the infant's self-organisation of postural competences by proposing varied sensorimotor experiences in a context supporting parent-child interaction. ${ }^{11}$ Physical therapists participating in the study followed a sensorimotor stimulation programme course and obtained a certification before coaching the parents. The parents are trained by a physical therapist with a doll and their infant, and they receive a booklet with step-by-step written descriptions and photographic illustrations. They administer the programme at home once a day between 37 weeks' gestation and 6 months of corrected age. A structured monthly diary is provided to the parents to keep data on number of administered sessions. During the 6-month stimulation period, the physical therapist meets the parents seven times for coaching and monitoring, and substantiates that the parents continue to use the outlined protocol. The parents may elect to discontinue the intervention at any time; this would cause the child to leave the study.

Parents also report eventual adverse incidents possibly related to the intervention. An observation diary will allow us to get compliance and safety data. During home visits, the physiotherapist assesses the stimulation performed by the parent.

The programme is divided in two phases to adapt the stimulation to the infant's neurological development, phase I from 37 weeks to $4 \frac{1}{2}$ months of corrected age and phase II from 41/2 to 6 months of corrected age. Each stimulation session lasts for $10 \mathrm{~min}$. It is performed when the infant is awake and quiet.

\section{Outcomes}

The study primary outcome is the BSID-III motor composite score at 24 months of corrected age. The secondary outcomes are BSID-III cognitive and language composite scores at this age and receptive and expressive communication and gross and fine motor scale scores at 6,12 and 18 months of corrected age. BSID-III is being done as described at 6,12, 18 and 24 months of corrected age in order to identify eventual developmental delays through the use of norm-referenced scores and to monitor infants' progress during intervention. The short form of PSI, ${ }^{16}$ which is also a secondary outcome, is administered at the same time points as the BSID-III in order to document time-related trends in the parent's relationship with the child. In addition, an exploratory outcome is the neurophysiological maturation evaluated using HD-EEG and recording of event-related potentials (ERPs) at term age, 3, 6, 12, 18 and 24 months of corrected age because it is anticipated that neurophysiological developmental changes might be age-specific over shorter periods in earlier than later infancy as assumed from general knowledge about brain maturation. Analysis of HD EEG signals will include frequency, power and synchrony of oscillation in resting state with a focus on spatial patterning using EEGLab and in-house complementary programme. Spectral content of oscillatory activity in evoked potentials will be used to study the dynamic brain processing underlying the different components of the evoked potentials, including (1) power perturbation in specific frequencies of ongoing brain oscillations induced by a stimulus, that is, event-related spectral perturbation; and (2) synchronisation of ongoing brain oscillations induced by a stimulus, that is, intertrial coherence. Source localisation will be performed using inverse modelling. Evaluations are performed in control and stimulation groups.

\section{Sample size calculation}

The sample size was calculated to detect a minimum clinically important difference in the BSID-III at 24 months of $1 \mathrm{SD}$ (15 points) with a power of $90 \%$ and using a level of significance of 0.05 (alpha value). This calculation indicates that 30 infants are needed in each group. Because of withdrawal risk, the target size is increased by $15 \%$ and 35 children will be included in each group.

\section{Allocation}

After parental consent is obtained, each participant is randomised into the experimental or comparison group. Web-based blocked randomisation list is created for each study site. Block size is fixed to 10. Blocks randomisation is used to achieve balance in the allocation of participants in the two arms (stimulation and control group). In case of multiple births, infants are randomised in the same group. Randomisation lists are accessible only to the sponsor representative not involved in the study management.

\section{Assessments}

All infants (in both groups) undergo systematic monitoring recommended and sponsored by the Belgian health authority for children born before 32 weeks' gestation or with a birth weight $<1500 \mathrm{~g} .{ }^{17}$ It includes a medical and neurodevelopmental evaluation performed by a paediatric neurologist, a psychologist and a physical therapist at the following time points: $4 \pm 1$ months, $11 \pm 2$ months and $231 / 2 \pm 1 \frac{1}{2}$ months of corrected age. In the 
settings where the study is conducted, the formal testing is performed using BSID-III.

\section{Bayley Scales of Infant Development III}

BSID-III assesses the developmental functioning of infants in order to identify suspected developmental delays through the use of norm-referenced scores and to monitor infants' progress during intervention. Cognitive, language and motor scales are performed.

All infants are assessed by a psychologist with Bayley III certification and blinded to the group distribution. BSID-III assessments are performed by the same assessor for the same child.

\section{Parental Stress index}

The short-form questionnaire of the $\mathrm{PSI}^{16}$ consists of 36 items divided into three subscales of 12 items each: parental distress, parent-child dysfunctional interaction and difficult child.

It is administered by the psychologist with the parent who declares himself to be the main provider of care. The psychologist is blinded to the group distribution.

\section{Electrophysiological assessment}

HD-EEG (64 or 128 electrodes depending on the head circumference, ActiveTwo, BioSemi, Amsterdam, The Netherlands) is recorded at term age, 3, 6, 12, 18 and 24 months of corrected age in a quiet environment. Background electroencephalographic recording is made during $10 \mathrm{~min}$.

Somatosensory stimulation electrodes are placed on the skin at the level of the ankle (posterior tibial nerve) and the intensity of somatosensory stimulation giving rise to a motor response is determined. Frequency stimulation is $0.5 \mathrm{~Hz}$. Flash visual stimulation is performed at the frequency of 1 and $9 \mathrm{~Hz}$. The use of a slow then fast visual stimulation frequency was determined to record 'transient' and type 'steady-state' visual evoked potentials respectively. Auditory stimulation is performed by using close but distinct synthetic phonemes 'da short' (standard stimulus $85 \%$ ) and 'da long' (deviant stimulus 15\%). EEG recording duration during somatosensory stimulation, visual and auditory stimulation is determined to get 200-300 events to study the ERPs.

\section{Data management}

All data are entered by investigators on paper case report form. Coded data are transmitted to the data manager who encodes them in the database. Consistency checks are performed against data already stored in the database. Access to the study data is restricted (locked rooms, passwords). Backup of the database is performed regularly.

The baseline characteristics will be compared between the two groups. For quantitative measurements, parametric or non-parametric tests will be used according to the distribution of the data. Categorical data will be compared by $\chi^{2}$ test and multivariate analysis. Perinatal, clinical neurodevelopmental, socio-demographic and neuroimaging data will be used for adjustment. Infants who are part of higher-order pregnancies will be included in the same arm but will contribute as individual components. Concerning the PSI, the results are specific for each child as this tool evaluates the tension experienced by the parent in the relationship with the child. For family variables (family structure, maternal age at birth, education of the primary caregiver, occupation and status of the primary income earner and language spoken at home), a sensitivity analysis including one child per higher-order pregnancy will be performed.

\section{Ethics and dissemination}

The study was approved by the Ethics Committees of the Hôpital Universitaire des Enfants Reine Fabiola and CHU Saint-Pierre. Dissemination of results will be made through reports written for parents, healthcare providers and policymakers. Scientific papers will be written and submitted to peer-review journals.

Collaborators Ana Maria Cebolla Alvarez, Carlos Cevallos Barragan, Guy Cheron, Bernard Dan, Jonathan Dan, Irit Daniel, Laurence Dewulf, Karin Duponcelle, Erell Filloque, Aleksandra Gajewska, Anne-Britt Johansson, Emmanuelle Jourdain, Marie Kennes, Emmanuelle Lempereur, Natan Napiórkowski , Karine Pelc, Catherine Preux, Marie Tackoen, Mireia Tremosa Ferrando, Fabienne Vanhorsigh, Inge Van Herreweghe, Bernard Wenderickx.

Contributors KP and BD conceived the study, coordinated its design and drafted the manuscript. ID and BW had significant input into the study protocol. All authors approved the final manuscript prior to submission.

Funding This work was supported by the Fondation Roger de Spoelberch, Fondation JED Belgique and the Fonds Iris Recherche managed by the King Baudouin Foundation.

Competing interests None declared.

Ethics approval Ethics committees of CHU Saint Pierre, Brussels, Belgium and Hôpital Universitaire des Enfants Reine Fabiola, Brussels, Belgium.

Provenance and peer review Not commissioned; externally peer reviewed.

Open Access This is an Open Access article distributed in accordance with the Creative Commons Attribution Non Commercial (CC BY-NC 4.0) license, which permits others to distribute, remix, adapt, build upon this work non-commercially, and license their derivative works on different terms, provided the original work is properly cited and the use is non-commercial. See: http://creativecommons.org/ licenses/by-nc/4.0/

(c) Article author(s) (or their employer(s) unless otherwise stated in the text of the article) 2017. All rights reserved. No commercial use is permitted unless otherwise expressly granted.

\section{REFERENCES}

1. Vohr BR, Wright LL, Poole WK, et al. Neurodevelopmental outcomes of extremely low birth weight infants $<32$ weeks' gestation between 1993 and 1998. Pediatrics 2005;116:635-43.

2. Spittle AJ, Spencer-Smith MM, Eeles AL, et al. Does the Bayley-III Motor Scale at 2 years predict motor outcome at 4 years in very preterm children? Dev Med Child Neurol 2013;55:448-52.

3. Linsell L, Malouf R, Morris J, et al. Prognostic factors for poor cognitive development in children born very preterm or with very low birth weight: a systematic review. JAMA Pediatr 2015;169:1162-72.

4. Linsell L, Malouf R, Morris J, et al. Prognostic factors for cerebral palsy and motor impairment in children born very preterm or very low birthweight: a systematic review. Dev Med Child Neurol 2016;58:554-69.

5. Linsell L, Malouf R, Johnson S, et al. Prognostic factors for behavioral problems and psychiatric disorders in children born very preterm or very low birth weight: a systematic review. J Dev BehavPediatr 2016;37:88-102. 
6. Hadders-Algra M. Early brain damage and the development of motor behavior in children: clues for therapeutic intervention? Neural Plast 2001;8:31-49.

7. Martin J, Friel K, Salimi I, et al. Corticospinal Development. Squire L, Encyclopedia of Neuroscience. Oxford: Academic Press, 2009:302-14.

8. Ismail FY, Fatemi A, Johnston MV. Cerebral plasticity: windows of opportunity in the developing brain. Eur J Paediatr Neurol 2017;21:23-48.

9. Spittle A, Orton J, Anderson PJ, et al. Early developmental intervention programmes provided post hospital discharge to prevent motor and cognitive impairment in preterm infants. Cochrane Database Syst Rev 2015;11:CD005495.

10. Musacchia G, Ortiz-Mantilla S, Realpe-Bonilla T, et al. Infant Auditory Processing and Event-related Brain Oscillations. J Vis Exp 2015;101:e52420.

11. Pelc K, Oberg GK, Vanhorsigh F, et al. Development of a parentaladministrered postural, movement and social interaction intervention programme to improve outcome in preterm infants: a feasibility study. Dev Med Child Neurol 2013;55:56.
12. Friel KM, Chakrabarty S, Martin JH. Pathophysiological mechanisms of impaired limb use and repair strategies for motor systems after unilateral injury of the developing brain. Dev Med Child Neurol 2013;55:27-31.

13. Roberts G, Howard K, Spittle AJ, et al. Rates of early intervention services in very preterm children with developmental disabilities at age 2 years. J Paediatr Child Health 2008;44:276-80.

14. Volpe JJ. Neurology of the newborn. 4th edn. London: W.B. Saunders, 2000

15. Woodward LJ, Anderson PJ, Austin NC, et al. Neonatal MRI to predict neurodevelopmental outcomes in preterm infants. $N$ Engl $J$ Med 2006;355:685-94.

16. Bigras M, Lafrenière PJ, Abidin RR. Indice de Stress Parental:manuel francophone en complément à l'édition américaine. Toronto: MultiHealth System, 1996.

17. INAMI. Enfants nés prématurés: intervention dans les frais des examens de suivi dans des centres spécialisés. http://www.riziv.f gov.be/SiteCollectionDocuments/convention_suivi_premature.docx (accessed 1 Aug 2017). 